\title{
A Wideband OFDM MIMO Measurement System for Antenna Evaluation
}

\author{
J. Mora-Cuevas, C. Gómez-Calero, L. Cuéllar, L. de Haro \\ Signals, Systems and Radiocommunications, Universidad Politécnica Madrid \\ E-mail: \{jmora,carlosgc,luiscn,leandro\}@gr.ssr.upm.es
}

\section{Introduction}

An OFDM-MIMO testbed has been developed based on a Software-Defined Radio (SDR) platform. It allows taking measurements in real scenarios where future wireless networks will be deployed. The novelty of this testbed is the bidimensional positioning system based on a planar scanner which was developed to control the position of the antenna array. The positioning system makes automatic testing possible reducing errors of the antenna array positioning. A set of software has been developed for making research works such as testing reconfigurable antennas, MIMO channel characterization and angular power profiles for proper spatial characterization in indoor and outdoor environments.

\section{General Description}

The wideband testbed consists of an $\mathrm{M}_{\mathrm{T}} \times \mathrm{M}_{\mathrm{R}}$ MIMO scheme, where $\mathrm{M}_{\mathrm{T}}$ and $\mathrm{M}_{\mathrm{R}}$ represent the number of transmitter and receiver antennas, respectively. The maximum number of antennas is 4 , and the operation frequency is $2.45 \mathrm{GHz}$, so antennas and channel characteristics for WLAN application can be measured. The testbed architecture can be divided into three main modules: signal processing, RF-IF and antenna module. The mode of operation is based on a post-processing of the received signal. The transmitted signals are made in a PC under Matlab environment where different OFDM signals are created for each transmitter antenna A complete description of the MIMO testbed can be found in [1].

The signals from PC are sent to the SDR platform which consists in 4292 Pentek boards. The signals are synchronously upconverted to IF ( $40 \mathrm{MHz})$ and sent to RF module, before being transmitted to antenna array. The RF module consists of four chains with plug and coaxial commercial components. The design has been carried out taking into account the required power levels to perform indoor/outdoor measurements. Regarding this, a power amplifier is situated in each RF chain to transmit up to $20 \mathrm{dBm}$ for each antenna

Fig. 1 shows the hardware scheme of the receiver, where the RF signals are amplified and downconverter to IF. Then, the signals are processed into the FPGAs and DSPs used in the SDR. In the FPGA board, both the synchronism stage and the FFT module have been carried out. Finally, baseband signals are received in the $\mathrm{PC}$ and stored. The receiver consist of two 6235 modules and one 4292 DSP board from Pentek. Each 6235 board implements in its FPGA the symbol synchronism stage for two RF chains. Time to frequency domain transformation has also been carried out into the FPGA at the FFT module. 


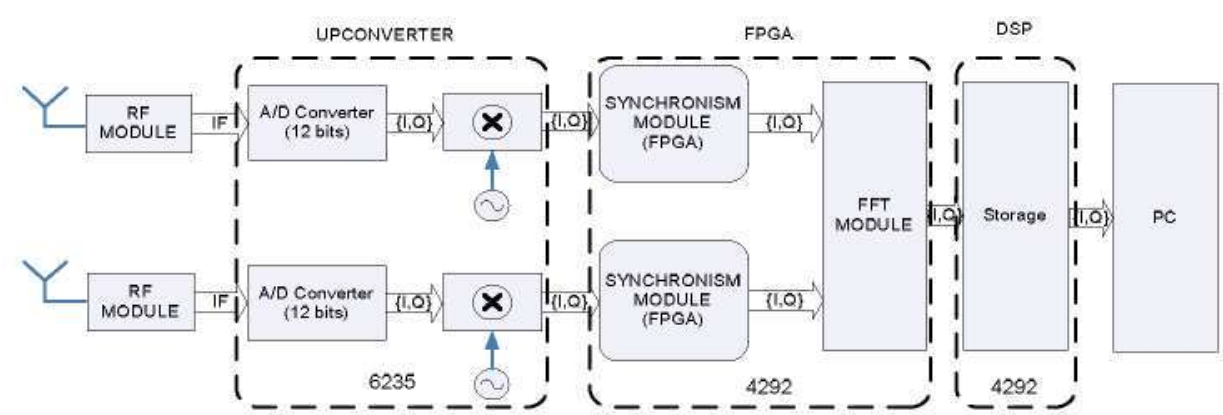

Fig. 1 Architecture of the OFDM-MIMO receiver

\section{MIMO-OFDM Configuration}

The OFDM modulation has been configured with a sample frequency (Fs) of 6.25 $\mathrm{MHz}$, an useful OFDM symbol length ( $\mathrm{Tu}=\mathrm{Ns} / \mathrm{Fs})$ of $163.84 \mu \mathrm{s}$, a guard interval $(\mathrm{Tg}=\mathrm{Ts} / 8)$ of $40.96 \mu \mathrm{s}$, an OFDM Symbol length (Ts) equal to $184.32 \mu \mathrm{s}$, with a subcarrier spacing $(1 / \mathrm{Tu})$ of $6.1 \mathrm{kHz}, 768$ subcarriers (Ns) and a bandwidth of $4687.5 \mathrm{kHz}$. As probe signal an OFDM symbol was generated and transmitted continuously. The number of carriers was 1024 from which 128 were null carriers put before and after the 768 subcarriers. At the beginning of the frame, a guard interval of length 128 was appended before the reference symbol. The null symbol is added and transmitted in order to avoid intersymbol interference (ISI) in the communication system. The structure of the OFDM symbol can be visualized in the Fig. 2.

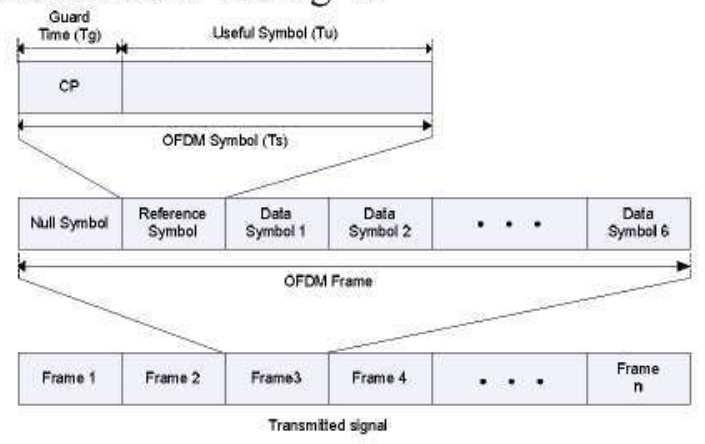

Fig. 2 OFDM frame structure.

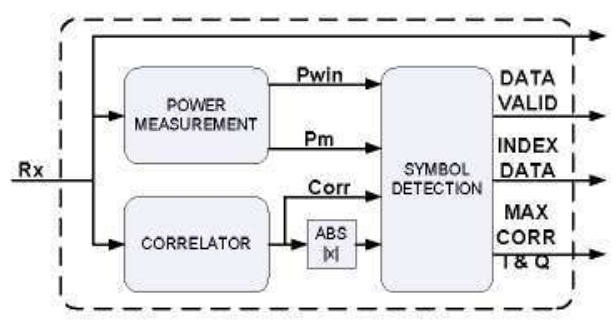

Fig. 3 Synchronism module.

For time synchronization with the reference symbol and carrier frequency error estimation, we applied the autocorrelation scheme of the cyclic prefix [2]. The synchronism module, Fig. 3, consists of a power measurement block, a correlation block and a symbol detector. The aim of the synchronism module is to determine the beginning of the OFDM symbol next to the null symbol inside of the same frame. The output contains the interval of samples where the reference symbol is, the index indicating the beginning of the reference symbol and the maximum correlation values. In Fig. 4, the peaks of the correlation output are at the beginning of each OFDM symbol within the frame. One frame of eigth symbols can be viewed at the center of the figure. Then, the index of the maximum peak passes through the FFT module to extract the OFDM information after removing the guard time. The received OFDM spectrum for a $2 \times 2$ OFDM-MIMO is 
depicted in Fig. 5. Then, the MIMO channel matrix is estimated in the PC in offline mode with the stored received signal.

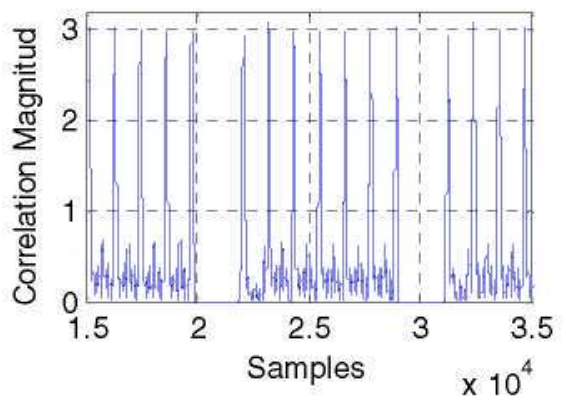

Fig. 4 Correlation Module.

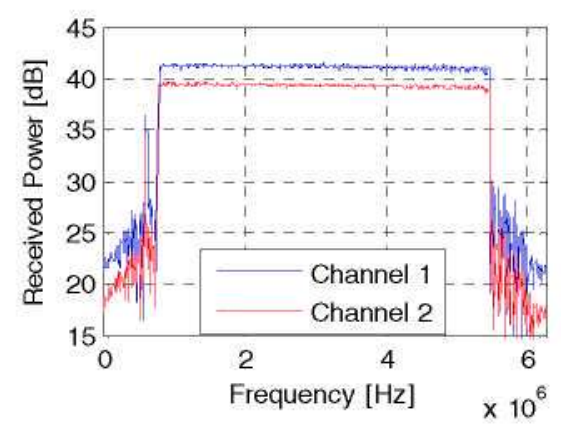

Fig. 5 Spectrum of received OFDM symbol.

\section{Array Positioning System}

It is well known that multipath scenarios cause fading in the radio channel link. In rich multipath scenarios like indoor environments, the fading becomes significant in distances on the order of wavelengths. Future wireless networks will be deployed in those scenarios being needed the proper spatial characterization of the radio channel.

For that purpose, we have design and implement a flexible antenna positioning system which is based on a planar scanner shown in Fig. 6. The position of the antenna support is controlled by two step-by-step motors which allow a translation movement in steps of $2.7 \mathrm{~mm}$. The system has two branches along two orthogonal axes which can move independently to build any bi-dimensional virtual array geometry. The length of both $\mathrm{x}$-axis and $\mathrm{y}$-axis branches is $1 \mathrm{~m}$. Thus, several array geometries can be formed and tested for studying spatial characteristics of the mobile radio channel (Angular Power Spectrum (APS), Direction-of-Arrival (DoA) and Angle-of-Arrival (AoA) profiles) as well as system parameters (capacity). For a multipath environment, ec. 1 and 2 represents the received data and the channel response respectively [3].

$$
\begin{gathered}
x^{l}(t)=\sum_{n=1}^{N} s_{n} h^{l}(t-n T) \\
h^{l}(t)=\sum_{d=1}^{D} a\left(\varphi_{d}, \theta_{d}\right) \cdot b_{d}^{l} \cdot g\left(t-\tau_{d}\right)
\end{gathered}
$$

where each d distinct propagation path is characterized by its DoA $(\varphi, \theta)$, path delay $\tau$ and complex path attenuation (fading) $b^{l}$ for every $l$ observation of the channel. Fig. 7 depicts the DoA profile for one signal source. The positioning system provides two possibilities for the antenna evaluation. The first one consists of mounting one antenna element in each scanner position and shifting along the linear axes to avoid the effect of the mutual coupling. The second option mounts an antenna array on the antenna support and considers a coupling factor in the data model. The OFDM-MIMO system supports up to 4 antenna ports in both transmitter and receiver side. 


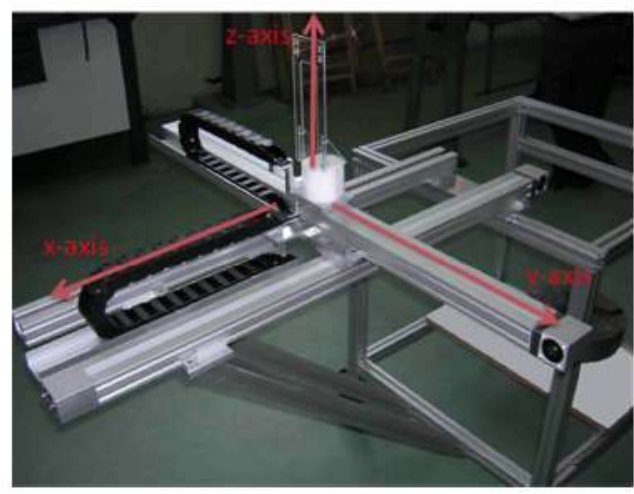

Fig. 6 Antenna positioning system.

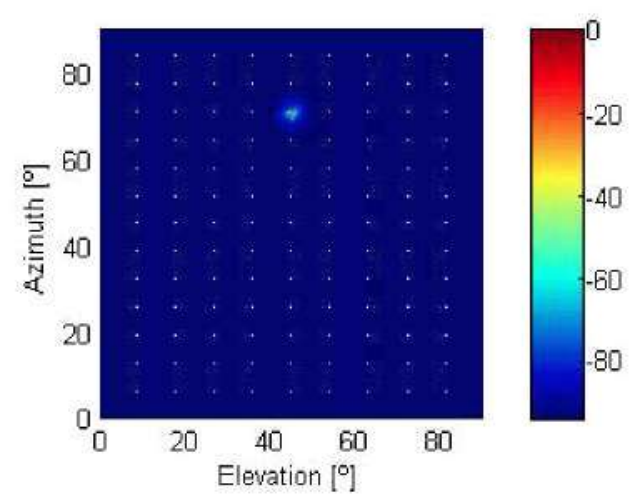

Fig. 7 Bi-dimensional DoA estimation.

The translation movement is controlled by software. A Unit Control (GCD) receives commands from a $\mathrm{PC}$ which are connected via a serial port. Thus, the errors due to mismatch in antenna spacing can be neglected since position is mechanically controlled. Also, the automatic repeatability of the measurement process causes a closer estimation of the sampled covariance matrix to the real covariance matrix. A user-friendly graphical interface has been designed in order to facilitate measurements analysis. This software implements a set of array geometries for analyzing its effect in the angular power spectrum and into the MIMO channel characterization for capacity analysis.

\section{Conclusions and future work}

An OFDM-MIMO testbed based on a SDR platform with very flexible antenna positioning system has been designed and presented for WLAN applications. Antenna positioning allows the proper spatial characterization of radio channel links in real scenarios where future wireless networks will be deployed. Some measurements in real indoor scenarios proving reconfigurable antennas are been carried out leading to some interesting results that will be presented in the conference.

\section{Acknowledgements}

One of the authors would like to thank Mexico's National Council of Science and Technology (CONACyT) for the support received to make this work.

\section{References}

[1] Carlos Gómez-Calero, Laura García-García, Leandro de Haro-Ariet, "New test-bed for evaluation of antenna and system performance for MIMO systems", Proceedings of EuCAP 2006, Nice, France, November 2006.

[2] J. J. van de Beek, M. Sandell, and P. O. Börjesson, "ML Estimation of Timing and Frequency Offset in OFDM".

[3] J.C. Liberti, T.S. Rappaport, Smart Antennas for Wireless Communications: IS-95 and Third Generation CDMA Application. Prentice Hall Commun. Engineering and Emerging Technologies Series. 